\title{
Front Matter: Volume 8293
}

, "Front Matter: Volume 8293," Proc. SPIE 8293, Image Quality and System Performance IX, 829301 (24 January 2012); doi: 10.1117/12.923968

SPIE Event: IS\&T/SPIE Electronic Imaging, 2012, Burlingame, California, United SPIE. States 


\title{
PROCEEDINGS IS\&T/SPIE Electronic Imaging \\ Science and Technology
}

\section{Image Quality and System Performance IX}

\author{
Frans Gaykema \\ Peter D. Bums \\ Editors
}

24-26 J anuary 2012

Burlingame, Califomia, United States

Sponsored and Published by

IS\&T-The Society for Imaging Science and Technology

SPIE 
The papers included in this volume were part of the technical conference cited on the cover and title page. Papers were selected and subject to review by the editors and conference program committee. Some conference presentations may not be available for publication. The papers published in these proceedings reflect the work and thoughts of the authors and a re published herein as submitted. The publishers are not responsible for the validity of the information or for any outcomes resulting from reliance thereon.

Please use the following format to cite material from this book:

Author(s), "Title of Paper," in Image Quality and System Performance IX, edited by Frans Gaykema, Peter D. Bums, Proc eed ings of SPIE-IS\&TElec tronic Imaging, SPIE Vol. 8293, Artic le CID Number (2012).

ISSN 0277-786X

ISBN 9780819489401

Copublished by

SPIE

P.O. Box 10, Bellingham, Washington 98227-0010 USA

Telephone +1 3606763290 (Pac ific Time)· Fax +1 3606471445

SPIE.org

and

IS\&T-The Society for Imaging Science and Technology

7003 Kilworth Lane, Spring field, Virginia, 22151 USA Telephone +1 7036429090 (Eastem Time)· Fax +1 7036429094

imaging.org

Copyright (c) 2012, Society of Photo-Optical Instrumentation Engineers and The Society for Imaging Science and Technology.

Copying of material in this book for intemal or personal use, or for the intemal or personal use of specific clients, beyond the fair use provisions granted by the U.S. Copyright Law is authorized by the publishers subject to payment of copying fees. The Transactional Reporting Service base fee for this volume is $\$ 18.00$ per article (or portion thereof), which should be paid directly to the Copyright Clearance Center (CCC), 222 Rosewood Drive, Danvers, MA 01923. Payment may also be made electronically through CCC Online at copyright.com. Other copying for republic ation, resale, advertising or promotion, or a ny form of systematic or multiple reproduction of any material in this book is prohibited except with permission in writing from the publisher. The CCC fee code is $0277-786 \mathrm{X} / 12 / \$ 18.00$.

Printed in the United States of America.

Paper Numbering: Proceedings of SPIE follow an e-First public ation model, with papers published first online and then in print and on CD-ROM. Papers are published as they are submitted and meet publication criteria. A unique, consistent, pemanent citation identifier (CID) number is assigned to each article at the time of the first publication. Utilization of CIDs allows articles to be fully citable as soon as they are published online, and connects the same identifier to all online, print, and electronic versions of the public ation. SPIE uses a six-digit CID artic le numbering system in which:

- The first four digits correspond to the SPIE volume number.

- The last two digits indic a te publication order within the volume using a Base 36 numbering system employing both numerals and letters. These two-number sets start with 00,01,02,03,04,05,06, 07, $08,09,0 \mathrm{~A}, 0 \mathrm{~B} \ldots \mathrm{0Z}$, followed by 10-1Z, 20-2Z, etc.

The CID number appears on each page of the manuscript. The complete citation is used on the first page, and an abbreviated version on subsequent pages. Numbers in the index correspond to the last two digits of the six-digit CID number. 


\section{Contents}

vii Conference Committee

SESSION 1 IMAGE QUALITY AND MOBILE IMAGING I: JOINT SESSION WITH CONFERENCE 8299

829302 Development of the I3A CPIQ spatial metrics [8293-01]

D. Baxter, STMicroelectronics Ltd. (United Kingdom); F. Cao, DxO Labs (France); H. Eliasson, Sony Ericsson Mobile Communications (Sweden); J. Phillips, Eastman Kodak Co. (United States)

\section{SESSION 2 IMAGE QUALITY AND MOBILE IMAGING II: JOINT SESSION WITH CONFERENCE 8299}

829303 Calibration and adaptation of ISO visual noise for I3A's Camera Phone Image Quality initiative [8293-02]

D. J. Baxter, A. Murray, STMicroelectronics Ltd. (United Kingdom)

829304 An objective method of measuring texture preservation for camcorder performance evaluation [8293-03]

K. Zhu, Univ. of Konstanz (Germany); S. Li, Univ. of Surrey (United Kingdom); D. Saupe, Univ. of Konstanz (Germany)

829305 Improving texture loss measurement: spatial frequency response based on a colored target [8293-04]

U. Artmann, D. Wueller, Image Engineering GmbH \& Co. KG (Germany)

SESSION 3 IMAGE ACQUISITION PERFORMANCE: CHARACTERIZATION AND MEASUREMENT

829306 The uncertainty of scanner illumination II [8293-05]

C. Cui, Lexmark International, Inc. (United States)

829307 Measuring the modulation transfer function of image capture devices: what do the numbers really mean? [8293-06]

X. Zhang, Purdue Univ. (United States); T. Kashti, D. Kella, T. Frank, Hewlett-Packard Indigo Ltd. (Israel); D. Shaked, Hewlett-Packard Labs. Israel Ltd. (Israel); R. Ulichney, Hewlett-Packard Labs. (United States); M. Fischer, Hewlett-Packard Labs. Israel Ltd. (Israel); J. P. Allebach, Purdue Univ. (United States)

829308 A fast, automatic camera image stabilization benchmarking scheme [8293-07] J. Yu, S. Craver, Binghamton Univ. (United States)

SESSION 4 IMAGE PROCESSING PERFORMANCE: CHARACTERIZATION AND MEASUREMENT

8293 OA A sharpness measure on automatically selected edge segments [8293-09]

S. Corchs, F. Gasparini, F. Marini, R. Schettini, Univ. of Milano-Bicocca (Italy) 
8293 OB Selecting the proper window for SSIM [8293-10]

S. B. McFadden, P. A. S. Ward, Univ. of Waterloo (Canada)

8293 OC Measurement of texture loss for JPEG 2000 compression [8293-11]

P. D. Burns, Burns Digital Imaging (United States); D. Williams, Image Science Associates (United States)

8293 OD A no-reference image quality metric for blur and ringing effect based on a neural weighting scheme [8293-12]

A. Chetouani, Institut des Systèmes Intelligents et de Robotique, Univ. Pierre et Marie Curie (France); A. Beghdadi, L2TI, Univ. Paris XIII (France)

8293 OE Quantifying image distortion based on Gabor filter bank and multiple regression analysis [8293-36]

B. Ortiz-Jaramillo, Univ. Nacional de Colombia (Colombia) and Gent Univ. (Belgium); J. C. Garcia-Alvarez, Univ. Nacional de Colombia (Colombia); H. Führ, RWTH Aachen (Germany); G. Castellanos-Dominguez, Univ. Nacional de Colombia (Colombia); W. Philips, Gent Univ. (Belgium)

\section{SESSION $5 \quad$ IMAGE DISPLAY PERFORMANCE: COLOR ADJUSTMENT}

8293 OF Comparative performance analysis of two picture adjustment methods: HSV vs. Y $C_{b} C_{r}$ [8293-13]

R. Safaee-Rad, Qualcomm Canada Inc. (Canada); M. Aleksic, Qualcomm (United States)

8293 OG Evaluation of preferred lightness rescaling methods for colour reproduction [8293-14]

Y. Chang, London College of Communication (United Kingdom)

$8293 \mathrm{OH}$ Investigation into the impact of tone reproduction on the perceived image quality of fine art reproductions [8293-15]

S. Farnand, J. Jiang, Rochester Institute of Technology (United States); F. Frey, Harvard College Library (United States)

\section{SESSION 6 PERCEPTUAL IMAGE QUALITY EXPERIMENTATION}

8293 Ol The mobile image quality survey game [8293-16]

D. R. Rasmussen, Qi Analytics LLC (United States)

8293 OJ Evaluation of perceived image sharpness with changes in the displayed image size [8293-17]

J. Y. Park, S. Triantaphillidou, R. E. Jacobson, G. Gupta, Univ. of Westminster (United Kingdom)

8293 OK Towards a perceptual quality metric for computer-generated images [8293-18]

P. Boulenguez, B. Airieau, M.-C. Larabi, D. Meneveaux, XLIM-SIC Labs., Poitiers Univ. (France)

8293 OL Assessing product image quality for online shopping [8293-19]

A. Goswami, S. H. Chung, N. Chittar, A. Islam, eBay Inc. (United States) 
SESSION 7A VISUAL ATTENTION: TASK AND IMAGE QUALITY: JOINT SESSION WITH CONFERENCE 8291

8293 OM How do we watch images? A case of change detection and quality estimation (Best Student Paper Award) [8293-20]

J. Radun, T. Leisti, T. Virtanen, G. Nyman, Univ. of Helsinki (Finland)

8293 ON Measuring saliency in images: which experimental parameters for the assessment of image quality? [8293-21]

C. Fredembach, G. Woolfe, J. Wang, Canon Information Systems Research Australia Pty. Ltd. (Australia)

SESSION 7B VISUAL ATTENTION: TASK AND IMAGE QUALITY: JOINT SESSION WITH CONFERENCE 8291

$829300 \quad$ Investigations of the display white point on the perceived image quality [8293-23]

J. Jiang, F. M. Abed, Rochester Institute of Technology (United States)

\section{SESSION 8 IMAGE QUALITY IN PRINT}

8293 OP A method of detecting changes in image quality via sensing on customer documents [8293-24]

W. Wu, B. Xu, J. Handley, Xerox Corp. (United States)

$82930 Q \quad$ Print quality analysis for ink-saving algorithms [8293-25]

M. V. Ortiz Segovia, Purdue Univ. (United States) and Océ Print Logic Technologies (France); N. Bonnier, Océ Print Logic Technologies (France); J. P. Allebach, Purdue Univ. (United States)

8293 OR Masking mediated print defect visibility predictor [8293-26]

X. Jing, Purdue Univ. (United States); H. Nachlieli, D. Shaked, Hewlett-Packard Labs. Israel Ltd. (Israel); S. Shiffman, Hewlett-Packard Indigo Ltd. (Israel); J. P. Allebach, Purdue Univ. (United States)

8293 OS Psychophysical evaluation of banding visibility in the presence of print content [8293-27] J. Zhang, Purdue Univ. (United States); H. Nachlieli, D. Shaked, Hewlett-Packard Labs. Israel Ltd. (Israel); S. Shiffman, Hewlett-Packard Indigo Ltd. (Israel); J. P. Allebach, Purdue Univ. (United States)

\section{SESSION 9 SYSTEM PERFORMANCE: VIDEO}

8293 OT No-reference video quality assessment of H.264 video streams based on semantic saliency maps [8293-28]

H. Boujut, J. Benois-Pineau, T. Ahmed, Lab. Bordelais de Recherche en Informatique, CNRS, Univ. Bordeaux (France); O. Hadar, Ben Gurion Univ. of the Negev (Israel); P. Bonnet, Audemat Worldcast Systems (France)

8293 OU Linking quality assessment of free-viewpoint video objects up with algorithm development [8293-29]

S. Kepplinger, Ilmenau Univ. of Technology (Germany) 
8293 OV A perceptual optimization of H.264/AVC bit allocation at the frame and macroblock levels [8293-30]

M. Hrarti, XLim-SIC Lab., Univ of Poitiers (France) and GSCM-LRIT Lab., CNRS, Univ. MohamedV (Morocco); H. Saadane, XLim-SIC Lab., Univ of Poitiers (France) and Nantes Univ. (France); M.-C. Larabi, XLim-SIC Lab., Univ of Poitiers (France); A. Tamtaoui, National Institute of Post and Telecommunications (Morocco); D. Aboutajdine, GSCM-LRIT Lab., CNRS, Univ. MohamedV (Morocco)

8293 OW QoE assessment method for mobile video services based on user motivation [8293-31]

F. Kobayashi, M. Masuda, T. Hayashi, NTT Corp. (Japan)

\section{SESSION 10 IMAGE QUALITY EVALUATION: NEW DEVELOPMENTS}

8293 OY A learning-based approach for automated quality assessment of computer-rendered images [8293-33]

X. Zhang, G. Agam, Illinois Institute of Technology (United States)

$82930 Z$ A comparison of techniques for super-resolution evaluation [8293-34]

M. Trifas, J. Straub, Jacksonville State Univ. (United States)

829310 Detection of image quality metamers based on the metric for unified image quality [8293-35]

K. Miyata, National Museum of Japanese History (Japan); N. Tsumura, Chiba Univ. (Japan)

\section{INTERACTIVE PAPER SESSION}

829311 Optimal patch code design via device characterization [8293-37]

W. Wu, E. N. Dalal, Xerox Corp. (United States)

829312 Influence of viewing device and soundtrack in HDTV on subjective video quality [8293-38] A. Redl, C. Keimel, K. Diepold, Technische Univ. München (Germany)

829313 Influence of viewing experience and stabilization phase in subjective video testing [8293-39]

C. Keimel, A. Redl, K. Diepold, Technische Univ. München (Germany)

829314 Perceptual image quality assessment metric that handles arbitrary motion blur [8293-40]

F. Gavant, L. Alacoque, A. Dupret, T. Ho-Phuoc, D. David, CEA-LETI Minatec(France)

829315 A unified method for comparison of algorithms of saliency extraction [8293-41]

T. Ho-Phuoc, L. Alacoque, A. Dupret, CEA-LETI Minatec (France); A. Guérin-Dugué,

GIPSA-Lab. (France); A. Verdant, CEA-LETI Minatec (France)

Author Index 


\title{
Conference Committee
}

\author{
Symposium Chairs
}

Majid Rabbani, Eastman Kodak Company (United States)

Gaurav Sharma, University of Rochester (United States)

Conference Chairs

Frans Gaykema, Océ Technologies B.V. (Netherlands)

Peter D. Burns, Burns Digital Imaging (United States)

Program Committee

Majed Chambah, Université de Reims Champagne-Ardenne (France)

Luke C. Cui, Lexmark International, Inc. (United States)

Mark D. Fairchild, Rochester Institute of Technology (United States)

Susan P. Farnand, Rochester Institute of Technology (United States)

Dirk W. Hertel, E Ink Corporation (United States)

Robin B. Jenkin, Aptina Imaging Corporation (United States)

Sang Ho Kim, Samsung Electronics Company, Ltd. (Korea, Republic of)

Mohamed-Chaker Larabi, Université de Poitiers (France)

Lindsay W. MacDonald, London College of Communication (United Kingdom)

Yoichi Miyake, Chiba University (Japan)

Göte S. Nyman, University of Helsinki (Finland)

D. René Rasmussen, Qi Analytics LLC (United States)

Sophie Triantaphillidou, University of Westminster (United Kingdom)

Eric K. Zeise, Kodak's Graphic Communications Group (United States)

Hongqin Zhang, Apple Inc. (United States)

\section{Session Chairs}

$1 \quad$ Image Quality and Mobile Imaging I: Joint Session with Conference 8299

Peter D. Burns, Burns Digital Imaging (United States)

Sebastiano Battiato, Università degli Studi di Catania (Italy)

$2 \quad$ Image Quality and Mobile Imaging II: Joint Session with Conference 8299

Peter D. Burns, Burns Digital Imaging (United States)

Sebastiano Battiato, Università degli Studi di Catania (Italy) 
3 Image Acquisition Performance: Characterization and Measurement Robin B. Jenkin, Aptina Imaging Corporation (United States)

$4 \quad$ Image Processing Performance: Characterization and Measurement Sang Ho Kim, Samsung Electronics Company, Ltd. (Korea, Republic of)

$5 \quad$ Image Display Performance: Color Adjustment

Sophie Triantaphillidou, University of Westminster (United Kingdom)

6 Perceptual Image Quality Experimentation

Mohamed-Chaker Larabi, Université de Poitiers (France)

7A Visual Attention: Task and Image Quality: Joint Session with Conference 8291

Bernice E. Rogowitz, Visual Perspectives Consulting (United States)

7B Visual Attention: Task and Image Quality: Joint Session with Conference 8291

Susan P. Farnand, Rochester Institute of Technology (United States)

$8 \quad$ Image Quality in Print

Frans Gaykema, Océ Technologies B.V. (Netherlands)

9 System Performance: Video

Luke C. Cui, Lexmark International, Inc. (United States)

10 Image Quality Evaluation: New Developments

Göte S. Nyman, University of Helsinki (Finland) 